\title{
Formación de especialistas en Oncología Radioterápica en España
}

\author{
V. Pedraza Muriel
}

\section{Introducción}

\subsection{Antecedentes}

En 2002, el Pleno del Consejo Nacional de Especialidades Médicas decidió revisar los objetivos, contenidos y métodos de los programas formativos de las distintas especialidades y encargó a las respectivas comisiones nacionales la puesta al día de los mismos en el plazo de un año. La Comisión Nacional de Oncología Radioterápica, tomando en consideración los contenidos del programa anterior, los avances experimentados en los últimos años, las innovaciones introducidas en diferentes países y sus propios criterios, concluyó el encargo recibido en el plazo previsto y presentó ante la autoridad competente el nuevo Programa de Formación en Oncología Radioterápica, cuyas líneas generales se exponen a continuación.

\subsection{Objetivos}

El objetivo básico de la formación en oncología radioterápica es la adquisición de competencia profesional suficiente, entendida ésta como la capacidad de los médicos especialistas de ofrecer a los pacientes una atención médica de calidad y a la sociedad un servicio eficiente a coste razonable. En España, el modelo educativo utilizado para la formación es el sistema de residencia basado en el autoaprendizaje y la adquisición progresiva de responsabilidades bajo la supervisión de uno o varios tutores en un centro asistencial acreditado para ello.

\subsection{Definición y ámbito de influencia de la especialidad}

La Oncología Radioterápica es una rama de la medicina clínica que utiliza la radiación ionizante, sola o en combinación con otras modalidades terapéuticas, para el tratamiento del cáncer y otras enfermedades no tumorales. La especiali-

Catedrático de Radiología y Medicina Física

Presidente de la Comisión Nacional de la Especialidad Granada dad está reconocida en España desde 1978 y con la denominación descrita desde 1984.

Los contenidos de la oncología radioterápica, sus fundamentos científicos y tecnológicos y su proyección clínica han experimentado en las últimas décadas un crecimiento y expansión considerables. El empirismo dominante en los años sesenta y setenta se ha transformado en un cuerpo doctrinal sólido, cuyas bases físicas y biológicas, combinadas con el desarrollo de la tecnología y la destreza clínica, configuran hoy una especialidad compleja de importancia fundamental en la investigación, diagnóstico y tratamiento de la enfermedad neoplásica y procesos no malignos.

Por otra parte, los avances experimentados en los campos de la cirugía, quimioterapia, hormonoterapia e inmunoterapia tumoral y el desarrollo de la biología molecular y de la radiobiología han introducido en el proceso formativo un conjunto de nociones -multidisciplinaridad, cooperación y formación continua- sin las cuales es imposible el ejercicio de la especialidad en niveles aceptables de calidad.

Para el tratamiento y cuidados generales de los pacientes de cáncer, los especialistas en oncología radioterápica deben poseer un conocimiento profundo de la enfermedad neoplásica y una sólida formación clínica. Son de su exclusiva competencia las indicaciones, planificación, ejecución y control de los tratamientos con radiación ionizante y terapéuticas afines, la evaluación de la respuesta y el seguimiento de los pacientes tratados y participan junto con otros especialistas en la prestación de cuidados paliativos y en la asistencia y apoyo clínico de los pacientes terminales.

Durante su etapa formativa, los especialistas en oncología radioterápica están obligados, además, a familiarizarse con los fundamentos del método científico, participar en programas y proyectos de investigación y adquirir la experiencia y el adiestramiento necesarios para alcanzar plena autonomía en los órdenes profesional, tecnológico y científico.

\subsection{Aspectos generales de la formación}

Objetivos de la formación, centros y unidades docentes acreditadas, desarrollo de los programas formativos y evaluación y control de la formación constituyen las etapas esenciales de un proceso educativo que exige el aprendizaje de conocimientos, la adquisición de habilidades y destrezas profesionales y la adopción por los especialistas en forma- 
ción de determinadas actitudes sobre la medicina en general y la oncología radioterápica en particular.

Los conocimientos a adquirir durante el período de residencia vienen especificados en los contenidos de la formación y en lo concerniente al método de aprendizaje es suficiente decir que las unidades y centros acreditados: i) deben ofrecer a los médicos en formación la oportunidad, el tiempo y los medios necesarios para que ellos mismos, con ayuda de otros, puedan acceder al cuerpo básico de conocimientos de la especialidad en el nivel adecuado; ii) deben supervisar y controlar dicho proceso.

El problema de las habilidades y actitudes es de importancia fundamental en la formación. Las que se refieren a la especialidad han de ser adquiridas en las unidades de formación correspondientes mediante el empleo de instrumentos educativos diversos: reflexión y esfuerzo personal, grupos de trabajo, talleres y otros. Las de orden general son competencia de los centros y deben constituir requisitos para la acreditación. A unas y otras nos referiremos posteriormente.

\subsection{Duración del período formativo: cinco años}

\subsection{Titulación previa: Licenciado en Medicina}

\section{Contenidos de la formación}

Los contenidos de la formación en oncología radioterápica, sumamente complejos y diversificados, pueden descomponerse en dos grandes grupos: i) contenidos básicos; ii) contenidos clínicos. Los primeros definen el sustrato científico de la especialidad. Los segundos delimitan su proyección profesional. Los contenidos básicos han de ser conocidos por los médicos especialistas en formación. Los contenidos clínicos han de ser conocidos y ejercitados por todos ellos. El centro de gravedad de la formación lo constituyen éstos últimos. Por esta razón, los conocimientos teóricos, aun siendo muy importantes, tienen carácter complementario con la formación clínica, que se considera fundamental. Brevemente expuestas, los contenidos de la formación se resumen en la forma siguiente:

\subsection{Historia natural y biología general de los tumores malignos}

- Historia natural de la enfermedad neoplásica.

- Carcinogénesis y desarrollo tumoral.

- Genética tumoral.

- Proliferación tumoral.

- Microambiente tumoral.

- Factores asociados con el desarrollo tumoral.

\subsection{Patología tumoral}

- Aspectos histopatológicos generales.

- Métodos diagnósticos.

- Cuadros anatomoclínicos.

- Criterios de clasificación general.

- Sistemas de estadificación tumoral.

\subsection{Radiobiología general}

- Transferencia de energía radiación-materia.
- Efectos generales de la radiación sobre los sistemas biológicos.

- Supervivencia y destrucción celular por radiación.

- Efectos celulares y tisulares de la radiación.

- Principios de protección radiológica.

\subsection{Radiobiología tumoral}

- Crecimiento y desarrollo tumoral.

- Respuesta tumoral a la radiación.

- Control tumoral por radiación.

- Respuesta a la radiación de los tejidos normales.

- Fraccionamiento de la dosis en radioterapia.

- Relaciones dosis-tiempo en la radioterapia fraccionada del cáncer.

- Ensayos predictivos en radioterapia.

- Bases biológicas de la reirradiación tumoral.

\subsection{Física y planificación en radioterapia}

- Radiofísica básica.

- Radiofísica aplicada a la radioterapia.

- Equipos y fuentes de radiación.

- Dosimetría física y clínica.

- Planificación geométrica y dosimétrica en radioterapia externa.

- Planificación en braquiterapia

- Garantía y control de calidad.

\subsection{Diagnóstico por la imagen}

- Métodos de diagnóstico.

- Semiología radiológica general.

- Aplicaciones en oncología y radioterapia.

- Técnicas de simulación.

- Nuevos avances.

- Fusión de imágenes.

\subsection{Tratamiento del cáncer: principios generales}

- Radioterapia externa y braquiterapia: fundamentos, metodología e instrumentación.

- Técnicas no convencionales en radioterapia y radioterapia especial.

- Cirugía oncológica: modalidades.

- Principios y modalidades de quimioterapia.

- Combinación de tratamientos.

- Criterios de respuesta al tratamiento y escalas de toxicidad.

- Factores generales de pronóstico.

- Tratamiento de soporte.

- Tratamiento de situaciones clínicas especiales.

- Aspectos psicosociales de la enfermedad neoplásica.

- Comités de tumores y grupos cooperativos.

\subsection{Localizaciones tumorales específicas: diagnóstico y tratamiento}

Epidemiología, historia natural, anatomía patológica, diagnóstico de extensión, factores de pronóstico, tratamiento, control local, supervivencia, toxicidad y complicaciones en las principales formas clínicas de cáncer. 


\subsection{Cuidados paliativos}

- Medicina paliativa y enfermedad terminal.

- Organización de los cuidados.

- Tratamiento de pacientes en situación terminal.

- Tratamiento del dolor.

\section{Rotaciones}

\subsection{Primer año}

Medicina Interna y/o especialidades médicas

Urgencias

Oncología Médica

Diagnóstico por la imagen

\subsection{Segundo año (primer semestre)}

\author{
Anatomía Patológica \\ Cirugía general \\ Ginecología \\ Hemato-oncología \\ Oncología pediátrica \\ Otorrinolaringología \\ Urología \\ Unidad de cuidados paliativos
}

\subsection{Segundo año (segundo semestre)}

Oncología Radioterápica

Tercero, cuarto y quinto año: Oncología Radioterápica

\subsection{Guardias}

Primer y segundo año: generales.

Tercer, cuarto y quinto año: específicas de especialidad.

\section{Metodología científica}

- Empirismo y experimentación.

- Medicina basada en la evidencia.

- Evaluación crítica de la literatura científica.

- Estudios clínicos observacionales.

- Protocolos de investigación.

- Estadística descriptiva e inferencial.

- Ensayos clínicos.

- Investigación de servicios.

\section{Gestion clínica en Oncología Radioterápica}

- Organización funcional de un servicio de radioterapia.

- Equipamiento básico y recursos humanos.

- Gestión de la actividad asistencial.

- Gestión económica.

- Algoritmos de decisión.

- Guías de práctica clínica.

\section{Bioética}

- Ética de la distribución de recursos en oncología radioterápica.

- Listas de espera.

- Eliminación de las desigualdades terapéuticas.

- Consentimiento informado.

- Confidencialidad y secreto profesional.

- Etica de las decisiones clínicas.

- Límites a la intensidad del tratamiento.

- Etica y deontología.

- Comités éticos.

\section{Habilidades y actitudes}

La formación en oncología radioterápica debe organizarse en las unidades docentes acreditadas de forma tal que, al final de su proceso educativo, los residentes de la especialidad hayan adquirido las habilidades y actitudes necesarias para el ejercicio profesional de la misma en grado aceptable. El conjunto de habilidades y actitudes generales exigible a todo especialista en Oncología Radioterápica se resume en la siguiente forma:

\subsection{Habilidades generales}

- Facilidad para la entrevista clínica.

- Competencia para la comunicación oral y escrita.

- Aptitud para el trabajo en equipo.

- Experiencia en el manejo de herramientas informáticas.

- Fluidez en la exposición oral con o sin ayuda de medios audiovisuales.

- Capacidad para la toma de decisiones.

- Identificación y evaluación correcta de síntomas y signos de enfermedad.

- Suficiencia en la metodología de la exploración física.

- Facultad de interpretación de pruebas complementarias.

- Pericia en la realización de técnicas quirúrgicas elementales.

- Soltura en la utilización de la tecnología propia de la especialidad.

- Capacidad para aplicar criterios de calidad en la práctica habitual.

\subsection{Habilidades específicas}

- Fijación de las indicaciones terapéuticas de la irradiación, sus objetivos generales y posible combinación con otros tratamientos.

- Elección del tipo de radiación utilizable en cada caso.

- Establecimiento de los parámetros básicos de la irradiación.

- Dominio de las técnicas de radioterapia externa y braquiterapia.

- Determinación de la eficacia biológica de los tratamientos fraccionados.

- Compensación en términos de dosis de las interrupciones terapéuticas.

- Control del funcionamiento operativo de las unidades de teleterapia.

- Elección de los volúmenes de irradiación pertinentes. 
- Planificación bi o tridimensional de los tratamientos con radioterapia.

- Análisis de resultados: control local, supervivencia y complicaciones.

- Establecimiento de límites de dosis en la exposición profesional a la radiación.

- Organización y dirección de una consulta de radio terapia.

- Determinación de los recursos de radioterapia necesarios por unidad de población.

- Evaluación de la eficacia y eficiencia de una insta lación de radioterapia.

\subsection{Actitudes}

- Percepción del carácter binomial -ciencia y profesiónde la medicina.

- Cooperación y abordaje multidisciplinar en el tratamiento de las diversas formas de cáncer.

- Contribución a la prevención primaria y secundaria, a la detección precoz y a la educación sanitaria de la población en materia de cáncer.

- Disponibilidad para el aprendizaje y la formación continua.

- Voluntad de asumir compromisos y responsabilida des.

- Conformidad con la noción de servicio hacia los pacientes y hacia la sociedad a que obliga el ejercicio de la medicina.

- Reconocimiento del derecho de los pacientes a una asistencia pronta y digna en condiciones de equidad con especial referencia al derecho de información.

- Conciencia de la repercusión económica de las decisiones.

- Preocupación por los aspectos deontológicos y éticos de la medicina en general y de la oncología radioterápica en particular.

- Aprendizaje de conceptos y métodos elementales de gestión.

- Rechazo de los conocimientos y prácticas clínicas no basadas en la evidencia científica.

- Adecuación profesional y personal al imperativo moral del trabajo bien hecho.

\section{Objetivos específico-operativos por año de residencia}

\subsection{Primer año}

- Establecimiento de relaciones con los pacientes.

- Adiestramiento en la realización de la historia clínica y la exploración física.

- Solicitud e interpretación de exámenes complementarios.

- Aprendizaje de la metodología general del trabajo asistencial.

- Formulación de juicios diagnósticos e indicaciones terapéuticas elementales.

- Guardias en medicina interna y urgencias.

- Conocimiento de los diferentes métodos de diagnóstico por la imagen.

- Aprendizaje de la anatomía y semiología radiológica básicas.
- Adiestramiento clínico general en el manejo de pacientes oncológicos

- Preparación suficiente en la utilización de fármacos.

- Adquisición de conocimientos y destreza clínica en la utilización de citostáticos.

- Conocimiento de medidas terapéuticas especiales en el tratamiento del cáncer.

- Asistencia a las actividades docentes de los servicios a los que sean adscritos.

\subsection{Segundo año}

- Adiestramiento clínico en especialidades relacionadas con la oncología

- Aprendizaje de los principios e indicaciones de la cirugía en patología tumoral.

- Práctica de exploraciones y/o técnicas especiales de interés oncológico.

- Formulación de elementos de pronóstico de orden general.

- Aprendizaje de los conceptos básicos de la oncología (carcinogénesis, historia natural, cinética de crecimiento, invasión tisular, capacidad metastatizante y otros).

- Conocimiento de los cuadros de presentación histopatológica de los tumores malignos.

- Conocimiento de los principios físicos y bases biológicas de la radioterapia.

- Participación en el desarrollo de experimentos simples relacionados con la física de la radiación (exposición, atenuación, distribución de la dosis) y la radiobiología tumoral (ensayos clonogénicos, curvas de supervivencia, coeficientes alfa y beta)

- Conocimiento y aprendizaje de los principios, objetivos y mecanismos operacionales de la protección radiológica.

- Asistencia y participación en las sesiones clínicas y actividades generales que se organicen por el centro asistencial y las unidades docentes respectivas.

- Asistencia a cursos formativos de carácter general y actividades relacionadas con la metodología del trabajo científico y la investigación biomédica en general.

\subsection{Tercer, cuarto y quinto año}

- Adquisición de los conocimientos, competencias, habilidades y actitudes en oncología radioterápica que se mencionan en los apartados 2, 4, 5 y 6 del presente programa.

- Inscripción y participación en un programa universitario de tercer ciclo, orientado hacia la obtención de la suficiencia investigadora $y$, en lo posible, el grado de doctor.

\section{Actividades generales}

\subsection{Asistenciales}

En el ejercicio de su función asistencial, las actividades de los MIR en formación deben ajustarse a los objetivos del plan de rotación antes descrito. Así, en su primer y segundo año de residencia, la formación clínica, estrictamente supervisa- 


\section{Pedraza Muriel}

da, debe enfocarse hacia el entrenamiento clínico de carácter general en las policlínicas o unidades hospitalarias mencionadas en dicho plan', metodología del trabajo asistencial, decisiones de hospitalización, indicaciones terapéuticas, utilización de fármacos (citostáticos, antibióticos, antiinflamatorios, esteroides, diuréticos, hipotensores, agentes osmóticamente activos), procedimientos quirúrgicos menores (incisiones, drenajes, biopsias, punciones, suturas), técnicas endoscópicas y exploraciones especiales (colonoscopia, tacto rectal, laringoscopia indirecta) ${ }^{2}$ y la participación en las actividades docentes y científicas generales de los servicios y/o especialidades en rotación ${ }^{3}$.

Durante su período de permanencia en Oncología Radioterápica (tercer, cuarto y quinto año de residencia) el proceso educativo de los MIR debe adquirir perfiles específicamente relacionados con la especialidad. En este sentido, las actividades formativas deben orientarse hacia el mejor conocimiento posible de la enfermedad neoplásica ${ }^{1}$, su origen, historia natural, mecanismos de crecimiento y extensión ', aspectos histopatológicos generales ${ }^{2,3}$, presentación clínica ${ }^{1}$, métodos de diagnóstico ${ }^{1,2}$, clasificación clínica y patológica ${ }^{1}$ 2 , respuesta a la radiación ${ }^{1}$, probabilidad de control ${ }^{2}$ y tratamiento aconsejable en los diferentes modelos y estadios evolutivos de cáncer ${ }^{2}$. La formación debe incluir, igualmente, un nivel de adiestramiento suficiente en dosimetría, simulación y planificación ${ }^{2}$, ejecución y control de los tratamientos con radioterapia externa ${ }^{1,2}$, braquiterapia ${ }^{1,2}$, técnicas especiales de irradiación ${ }^{2,3}$, tratamiento de síndromes de urgencia, cuidados paliativos y situaciones terminales ${ }^{1,2}$. Este conjunto de actividades debe desarrollarse, por otra parte, mediante la asunción progresiva de responsabilidades y la búsqueda por los residentes de autonomía funcional, bajo la supervisión de los médicos especialistas de la unidad docente respectiva.

\subsection{Docentes y científicas}

La formación asistencial será compatible, en todo caso, con el desarrollo de actividades docentes y científicas generales en el ámbito de la especialidad. Entre las primeras, deben considerarse obligatorias las siguientes: participación en sesiones clínicas (nuevos casos, pacientes con problemas específicos, indicaciones terapéuticas) ${ }^{1}$, sesiones bibliográficas $^{1}$, sesiones clínico-patológicas ${ }^{1,2}$, sesiones generales del hospital $^{2,3}$, sesiones monográficas ${ }^{2,3}$ y reuniones y debates en comités de tumores y unidades pluridisciplinares (mama, linfomas, cabeza y cuello, ginecología, tumores digestivos, oncología pediátrica y otras) 2, 3. Entre las segundas, cabe incluir la asistencia a cursos formativos de la especialidad y áreas afines ${ }^{1}$, la colaboración en el diseño y ejecución de ensayos clínicos y/o proyectos de investigación básica o aplicada', la publicación de trabajos científicos ${ }^{2}$, la presentación de comunicaciones y/o ponencias en congresos y reuniones médicas diversas (jornadas, workshops, conferencias, cursos avanzados) $)^{2}$, en los casos en los que ello sea posible, la realización de un programa de doctorado1,2. El aprendizaje del inglés ${ }^{1}$ se incluye, asimismo, en este segundo grupo de actividades.

\subsection{Niveles de responsabilidad}

Los niveles de responsabilidad de las actividades mencionadas se han especificado entre paréntesis para cada una de ellas con arreglo al siguiente código: actividades realizadas, directamente por el residente (1), actividades realizadas por el residente bajo la supervisión de un tutor (2), actividades realizadas por el personal sanitario del centro o servicio correspondientes, con la presencia y/o asistencia del residente (3).

\section{Propuestas adicionales}

Las unidades docentes acreditadas para la formación de médicos especialistas en Oncología Radioterápica deben asegurar el acceso de los mismos a laboratorios de biología tumoral durante un tiempo no inferior a tres meses y facilitar su asistencia a cursos nacionales o internacionales de radiobiología de nivel adecuado.

Se recomienda igualmente la participación de los especialistas en formación en cursos formativos de carácter básico o clínico, nacionales e internacionales, relacionados con la Oncología Radioterápica. Para añadir una dimensión europea a la formación se recomienda, como mínimo, la asistencia a dos cursos formativos de nivel europeo y la asistencia, al menos, a un congreso internacional o europeo de la especialidad. Todo ello con independencia de las actividades docentes y científicas especificadas en el presente programa.

Es importante, asimismo, que los especialistas en formación adquieran un aceptable nivel de formación en protección radiológica, conforme a la normativa vigente en nuestro país, y obtengan la licencia de supervisor de instalaciones radiactivas (especialidad en radioterapia) previa la superación del curso de capacitación correspondiente.

Durante su proceso formativo, los MIR en formación en Oncología Radioterápica deben disponer de tiempo suficiente para el estudio, la preparación de casos y el análisis de literatura científica. Dicho período de tiempo no debe ser, en ningún caso, inferior al diez por ciento del tiempo semanal efectivo dedicado a la formación.

Los especialistas en formación en Oncología Radioterápica deben tener un acceso privilegiado a la investigación básica y clínica, disponer de tiempo para ello, participar en los proyectos en desarrollo y adquirir la preparación y el adiestramiento necesarios para su formación científica.

En la medida de lo posible, los MIR de Oncología Radioterápica deberían poder realizar una parte de su proceso educativo en una unidad docente, nacional o internacional, distinta a aquella donde estén adscritos. El principio de movilidad subyacente a esta posibilidad se considera de gran importancia para el futuro de la especialidad.

\section{Evaluación}

Para el desarrollo del presente programa, se considera indispensable que las unidades docentes acreditadas dispongan de infraestructura, equipamiento y recursos humanos suficientes. Periódicamente, se valorarán la naturaleza y grado de utilización de los recursos disponibles de conformidad con los criterios establecidos al efecto.

El cumplimiento de las actividades incluidas en este programa es inexcusable para la consecución de los objetivos y fines de la formación. Se recomienda, por ello, la revisión periódica del grado de cumplimiento de los mismos median- 
te procedimientos distintos (informes de los tutores y/o responsables de las unidades docentes, encuestas, métodos de auditoría externa y otros).

Con el propósito de verificar la realización de los objetivos específico-operativos previstos en el programa formativo en los órdenes cognoscitivo, de habilidades y actitudes, se recomienda introducir en el proceso de formación el denominado Libro del Residente, en el que deben figurar, necesariamente, las actividades desarrolladas, los objetivos conseguidos y el grado de responsabilidad alcanzado por los médicos especialistas en formación.

La organización y desarrollo de una prueba de evaluación global, de carácter voluntario, al final del período de residencia, se considera, por último, un instrumento esencial para garantizar la calidad de la formación impartida.

\section{Responsabilidades formativas}

Se considera conveniente, por último, para la consecución de los objetivos del presente programa, que los centros y unidades docentes respectivas asuman ciertos compromisos educativos vinculables con la acreditación. Son importantes a este respecto los siguientes:

- En cada centro debe existir una estructura de dirección encargada de supervisar los diferentes programas formativos, atribuir responsabilidades a nivel de unidad, proponer planes de desarrollo, identificar problemas, alumbrar soluciones y responder ante las autoridades sanitarias del desarrollo general de la docencia postgraduada en la institución.

- En cada unidad debe estar definida unipersonalmente la responsabilidad de la organización y cumplimiento del programa de la especialidad de que se trate. El responsable de la docencia debe tener autoridad delegada suficiente para planificar tareas, elegir a los tutores, programar actividades, encargar misiones, efectuar cambios, corregir desequilibrios e informar periódicamente a sus superiores del proceso formativo.

- Las acreditaciones de centros y unidades docentes deben otorgarse por períodos limitados de tiempo susceptibles o no de renovación en función de los resultados obtenidos.

- La multiplicidad y complejidad de las funciones inherentes a la formación especializada exigen, por último, una especial aptitud y preparación de los que ejerzan como tutores. Condiciones necesarias para ello son, en principio, las siguientes: i) voluntad de participar en el proce- so educativo; ii) experiencia clínica contrastada; iii) suficiencia en la metodología docente y asistencial; iv) conocimiento del método científico. Paradigma del tutor ideal sería, por tanto, un especialista con diez o más años de experiencia profesional y título de doctor.

\section{Consideración final}

El programa formativo de la especialidad se ha elaborado tomando como referencia el actualmente vigente en los países de la Unión Europea. Por razones de homologación y convergencia y para facilitar la libre circulación de personas, bienes y servicios en el ámbito europeo, se ha propuesto elevar a cinco años la duración del proceso formativo por ser éste el período de tiempo que rige para la Oncología Radioterápica en la Unión. La naturaleza y extensión de los contenidos educativos, la complejidad de los procedimientos terapéuticos actualmente utilizados en el tratamiento del cáncer y de otras enfermedades con radiación ionizante y la adquisición de los conocimientos y competencias necesarios para el ejercicio profesional justifican, sobradamente, la duración propuesta. En esta hipótesis, el tiempo de formación mínimo en el ámbito específico de la Oncología Radioterápica debe ser igual o superior al sesenta por ciento (tres años) del tiempo global de formación antes descrito

\section{Equipo de trabajo}

El plan de formación descrito en los párrafos precedentes ha sido elaborado por los miembros de la Comisión Nacional de Oncología Radioterápica que se mencionan a continuación: Ignacio Azinovic, Pedro Bilbao, José Luis Carceller, Ricardo Escó, Eduardo Lanzós, Amalia Palacios, Vicente Pedraza, Ignacio Petschen y Reinerio Rodríguez.

\section{Bibliografía}

1. Baumann M, Leer JWH, Dahl O, et al. Updated European core curriculum for radiotherapists (radiation oncologists). Radiother Oncol 2004; 71:107-13.

2. Röttinger $E$, Barrett $A$, Leer JWH. Guidelines for the infrastructure of training institutes and teaching departments for radiotherapy in Europe. Radiother Oncol 2004; 71:123-4.

3. Coffey M, Degerfält J, Osztavics A, van Hedel J, Vandevelde G. Revised European core curriculum for RTs. Radiother Oncol 2004; 71:137-58. 